\title{
REQUISITOS DO PROJETO ELÉTRICO DE MOTORES DE INDUÇÃO PARA ACIONAMENTO
}

\section{POR VARIADOR DE VELOCIDADE}

\section{Resumo}

O acionamento de motores elétricos de indução por inversores estáticos de frequência (comercialmente denominados variadores de velocidade) é uma solução já amplamente utilizada na indústria. Contudo sendo, há ainda muito para ser, feito, estudado e compreendido principalmente ao nível da interação entre o variador $e$ o motor, para que tais aplicações sejam efetivamente vantajosas em termos de eficiência energética e atrativas em termos de custo. Neste artigo são expostos os principais desafios que são colocados aos fabricantes de motores e que resultam da alimentação dos mesmos por uma fonte de tensão PWM com elevadas frequências de comutação. São apresentadas algumas considerações normativas $e$ apresentadas algumas soluções comummente utilizadas para mitigar os problemas sentidos.

\section{Introdução}

De todos os tipos de motores o de indução é o mais usado, quer no sector industrial quer no sector comercial.

O seu baixo custo, uma simples e robusta construção, a elevada fiabilidade, a reduzida manutenção e os níveis de eficiência conseguidos com as atuais tecnologias de controlo fazem dele um elemento de conversão eletromecânica muito apetecível. Contudo, é já hoje unanimemente aceite que o desempenho da motor de indução é diferente quando este é alimentado por um conversor estático de potência (variador de velocidade), ou quando alimentado diretamente pela tensão da rede. Em aplicações onde o desempenho exigido não é elevado a utilização de motores com uma construção padrão tem resultados satisfatórios, mas quando é requerido elevado desempenho é mais apropriado um motor com uma conceção específica o que vai atenuar as restrições construtivas associadas às aplicações de velocidade constante.
Os variadores de velocidade podem utilizar um controlo escalar ou vetorial. Em ambos os casos a tensão que geram é do tipo PWM (Pulse Width Modulation) com um conteúdo harmónico e gradientes de tensão cujos efeitos no motor assumem grande relevância e que necessitam de ser considerados no projeto do motor. O efeito dos elevados gradiente de tensão é particularmente importante quando o motor é alimentado por cabos longos, pois a impedância dos cabos de alimentação reduz o gradiente de tensão aos terminais do motor mas cria aí um efeito de onda amortecida onde as sobretensões podem ser várias vezes superiores à tensão nominal, reduzindo a vida útil do isolamento dos enrolamentos do motor. Para mitigar este problema alguns autores defendem a utilização de filtros [1]. Os harmónicos de tensão e corrente apesar de não contribuírem para um aumento do binário motor, uma vez que não aumentam o fluxo fundamental no entreferro que gira à velocidade síncrona, aumentam as perdas no ferro e no cobre, respetivamente $[2,3,4]$. Para agravar o problema o acionamento a velocidades mais baixas reduz a ventilação do motor. A conjugação destes dois fatores trás problemas adicionais ao nível do isolamento dos enrolamentos, podendo mesmo ser excedido o limite de temperatura definido pela classe de isolamento com que o motor foi construído. Este aumento da temperatura pode não provocar danos imediatos mas provocará, com certeza, uma diminuição do tempo de vida do motor. Para a maioria dos atuais isolantes um aumento da temperatura de $10^{\circ} \mathrm{C}$ resultará numa redução em $50 \%$ no tempo de vida esperado do mesmo [5].

Para além doa efeitos anteriormente referidos, podem aparecer outros efeitos, que não se devem especificamente aos harmónicos, mas que são também relevantes e que não devem ser desprezados, tais como a circulação de corrente pelos rolamentos [6] e o aumento dos níveis de vibração e ruído $[7,8]$. 


\section{Considerações Normativas}

Analogamente a vários outros aspetos construtivos, também as condições de acionamento do motor de indução por variador de velocidade estão normalizadas.

Os principais organismos normalizadores internacionais dedicam vários capítulos das suas normas de máquinas elétricas rotativas inteiramente a esta temática, o que demonstra também a importância do tema. Assim, destacam-se:

- IEC: 60034-17: Rotating Electrical Machines - Cage induction motors when fed from converters application guide;

- IEC 60034-25: Rotating Electrical Machines - Guide for the design and performance of cage induction motors specifically designed for converter supply;

- NEMA MG1 - Part 30: Application considerations for constant speed motors used on a sinusoidal bus with harmonic content and general purpose motors used with adjustable-voltage or adjustable-frequency controls or both;

- NEMS MG1 - Part 31: Definite purpose inverter-fed polyphase motor.

\subsection{Conteúdo Harmónico}

Os valores limite de distorção harmónica da tensão gerada pelo variador de velocidade, e consequentemente da corrente, não estão normalizados. No entanto, as normas consideram o aumento das perdas do motor devido ao uso do variador.

A norma IEC 60034-17 exemplifica o aumento das perdas do motor devido ao uso de um variador com o caso prático de um motor com uma carcaça 315 , com valores nominais de binário e velocidade, apresentando para este caso perdas 15\% maiores, sendo a maior contribuição a das perdas no ferro com $12 \%$ desses quinze. No capítulo IEC 60034-17 é comparado o acionamento à carga nominal e em vazio de um motor de $37 \mathrm{~kW}$ alimentado com tensões a variar entre os 20 e os $100 \mathrm{~Hz}$, sendo apontadas soluções para mitigar o aumento de perdas verificado.
A NEMA MG1 - Parte 30 considera uma redução percentual do binário do motor (derating factor) para evitar o excessivo sobreaquecimento de um motor alimentado por um inversor, que estará sujeito a correntes harmónicas decorrentes do conteúdo harmónico da tensão PWM.

\subsection{Gradiente de tensão}

As definições do tempo de subida (rise time) são diferentes na norma IEC e NEMA, o que gera divergências de interpretação e conflitos entre fabricantes e consumidores.

Segundo o IEC 60034-25 o tempo de subida corresponde ao tempo que a tensão leva para subir de 10 a $90 \%$ da tensão do barramento DC. Pelo critério NEMA deve-se tomar o valor da tensão do barramento, enquanto que pelo IEC se deve usar o valor de pico da tensão aos terminais do motor. Este último leva em consideração o efeito do cabo de alimentação, podendo por isso ser mais preciso mas é mais difícil de ser previsto ou estimado a priori.

\subsection{Isolamento dos enrolamentos}

Relativamente aos efeitos sobre 0 isolamento dos enrolamentos dos motores, tanto a IEC 60034 como a NEMA MG1 apresentam tabelas e gráficos com os valores da tensão de pico e tempo de subida da tensão (rise time) que os isolamentos devem suportar. Para motores com tensões de alimentação mais elevadas sugerem o reforço do isolamento e a instalação de filtros na saída do inversor para limitar as tensões de pico. Garantindo que a tensão de saída do inversor não excede os limites apresentados na norma assume-se que não haverá significativa redução na vida útil do isolamento por stress de tensão.

\subsection{Correntes de circulação pelos rolamentos}

Os problemas devidos a tensão/corrente induzida no eixo são agravados pela forma de onda da tensão PWM gerada pelos variadores de velocidade, devido a estas serem tendencialmente desequilibradas e por terem componentes de alta frequência [9]. 
A IEC 60034 recomenda o uso de filtros para redução da componente de sequência zero, a redução do $d V / d t$ e o isolamento dos rolamentos em motores com carcaças acima da 315 e ou potências superiores a 400 kW. Recomenda ainda a utilização de escovas de aterramento no eixo.

A NEMA MG1 refere a maior ocorrência de correntes de circulação nos rolamentos em motores com carcaças mais pequenas, menores que 500. E sugere o isolamento de ambos os rolamentos, ou alternativamente a utilização de escovas de aterramento no eixo para desviar a corrente dos rolamentos.

\section{Considerações Relativas aos Harmónicos}

Tal como referido anteriormente o motor de indução acionado por um variador de velocidade recebe nos seus terminais uma tensão PWM, pelo que estará sujeito a harmónicos que afetam o seu desempenho, nomeadamente, um aumento de perdas e de temperatura de funcionamento. A influência do variador sobre o motor depende de uma série de fatores relacionados com o controlo, tais como a frequência de comutação, a largura efetiva dos pulsos, o número de pulsos, entre outros.

A Figura 1 ilustra a forma de onda típica da tensão composta e respetivo espectro de frequências de uma alimentação PWM. É visível o elevado conteúdo harmónico, com componentes até cerca dos $50 \mathrm{kHz}$.

Estas componentes harmónicas não contribuem para a produção de binário motor, uma vez que não aumentam o fluxo fundamental no entreferro, que gira à velocidade síncrona. Contudo, são responsáveis por um aumento das perdas, uma vez que para frequências mais elevadas as perdas por histerese aumentam, assim como aumenta a saturação efetiva do núcleo. Concomitantemente, as correntes harmónicas aumentam as perdas por efeito de Joule nos condutores.

A operação do motor a velocidades de rotação mais baixas promove uma redução na ventilação e consequente perda de transferência de calor (em motores autoventilados), contribuindo também para a elevação da temperatura de estabilização térmica.
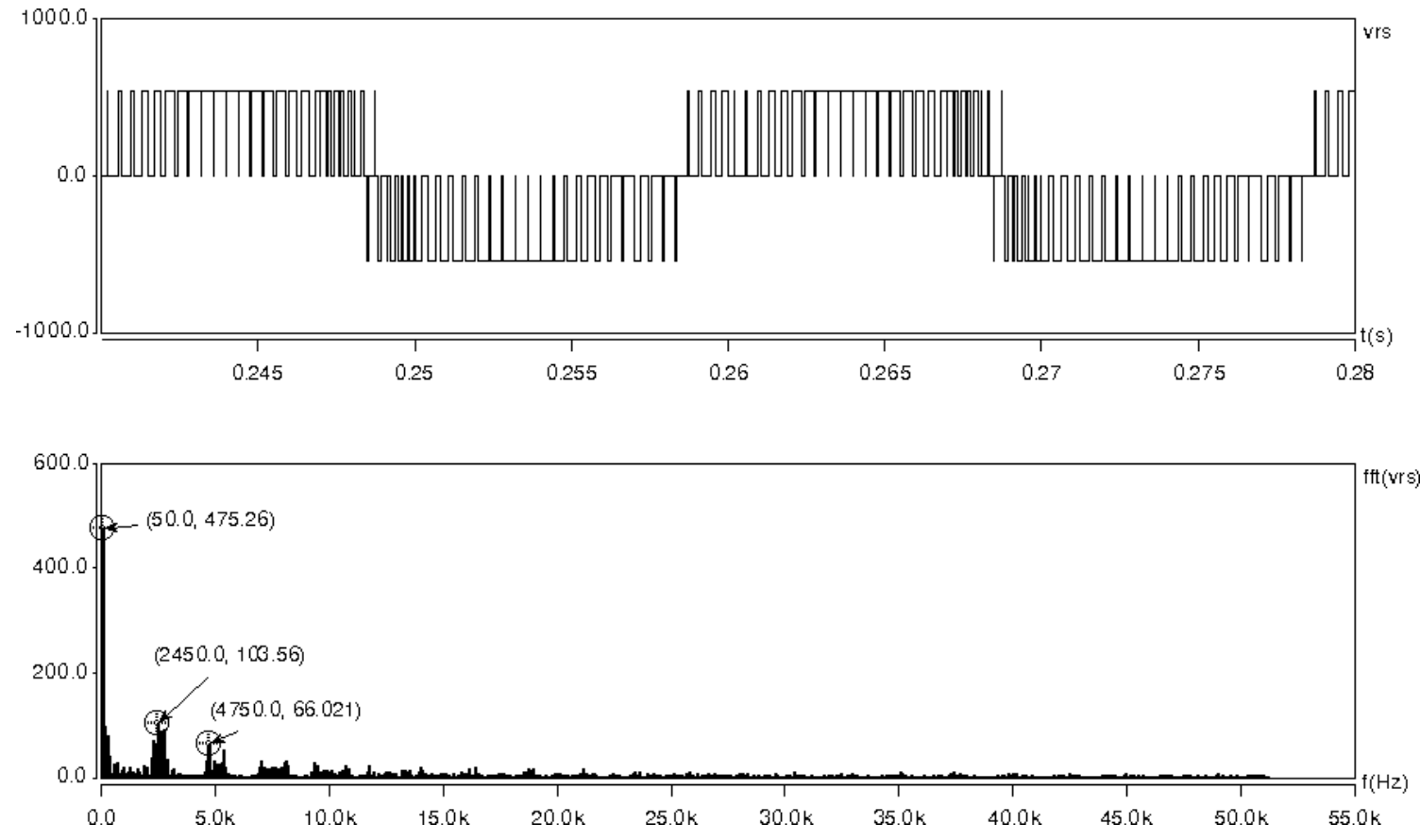

Figura 1. Tensão composta e respetivo espectro de frequências 
Portanto, quando da operação com variador de velocidade, e por efeito da influência conjunta dos fatores supracitados devem ser consideradas medidas para mitigação do sobreaquecimento do motor, nomeadamente:

- Redução do binário nominal (sobredimensionamento do motor);

- Utilização de um sistema de ventilação independente;

- Utilização do "fluxo ótimo" (solução patenteada pela WEG [4]).

$\mathrm{Na}$ Figura 2 é apresentada uma curva típica de sobredimensionamento aplicável a motores de produção em série com projeto padrão. .

Na Tabela 1 são apresentadas algumas medidas, genéricas, para mitigação do conteúdo harmónico da tensão gerada por variadores de velocidade.

Tabela 1. Métodos de redução de harmónicos da tensão gerada por variadores de velocidade (fonte [10])

\section{Método de redução de harmónicos}

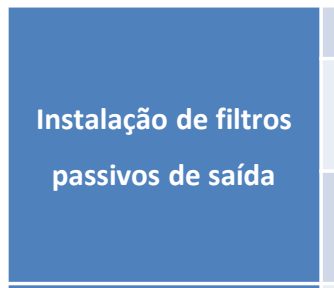

\section{Utilização de}

inversor com mais

níveis

Melhoria na

qualidade da

modulação

PWM

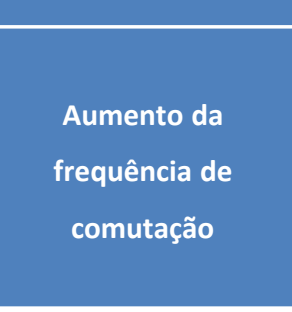

\section{Características da solução}

Aumento do custo da instalação

Restrições para operação nos modos vetoriais

Queda de tensão (redução da potencia do motor)

Aumento de custos

Redução de fiabilidade do inversor Aumento da complexidade do controlo

Space Vector Modulation

Não aumenta custos

Melhoria no controle de tensão

Maior rendimento do conjunto (motor + inversor)

Redução do rendimento do inversor (aumento das perdas por comutação)

Aumento das correntes de fuga para a terra

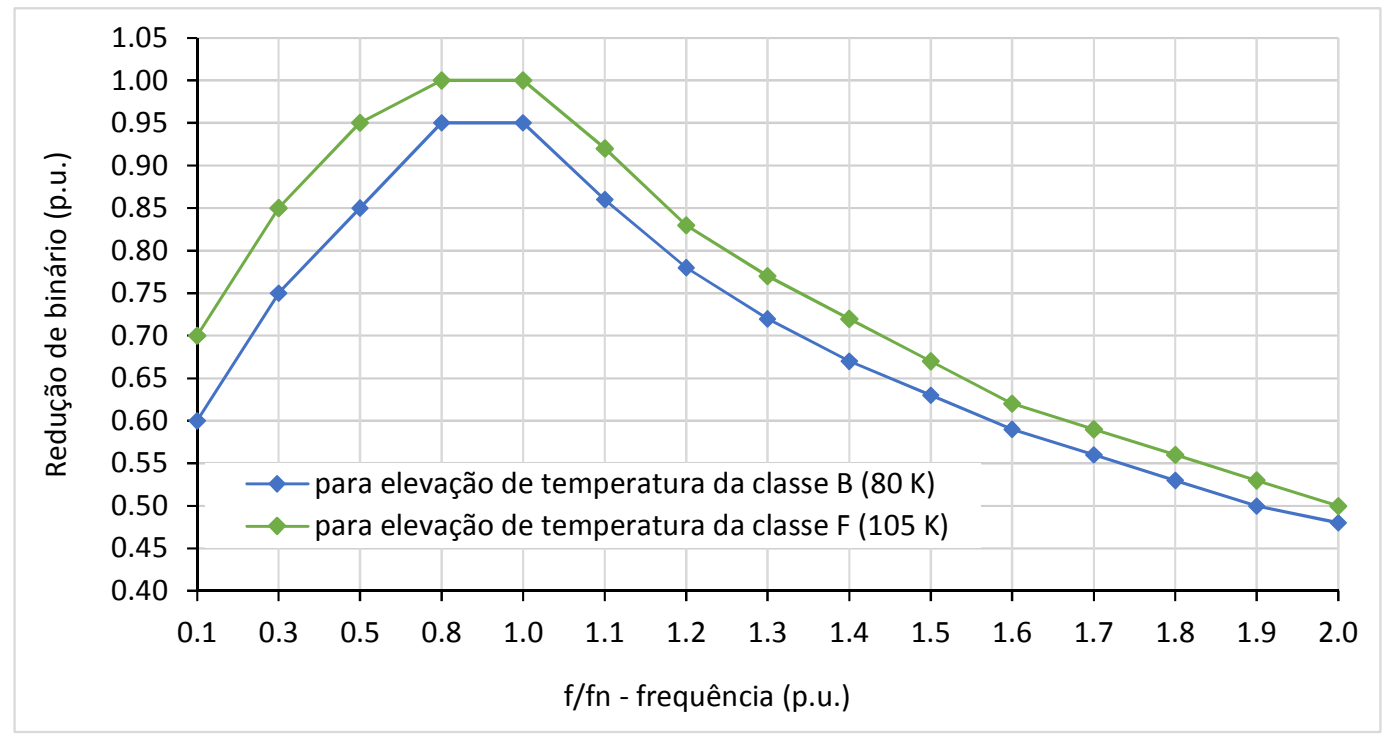

Figura 2. Curva típica de sobredimensionamento aplicável a motores de produção em série com projeto padrão 


\section{Considerações Relativas ao Isolamento dos Enrolamentos do Motor}

A evolução dos semicondutores de potência tem levado à criação de transístores de potência mais eficientes e mais rápidos. Consequentemente, os conversores eletrónicos de potência operam a frequências de comutação superiores o que tem vantagens. Contudo, do ponto de vista do motor acarretam algumas consequências indesejáveis, tais como o aumento da emissão eletromagnética e a provável incidência de picos de tensão, bem como elevados valores de $\mathrm{dV} / \mathrm{dt}$ (taxa de variação da tensão no tempo) aos terminais dos motores alimentados por variadores de velocidade.

Dependendo das características do controlo, da modulação PWM adotada, e da impedância do cabo e do motor, podemse gerar sobretensões, repetitivas, aos terminais do motor. Estes pulsos de tensão podem reduzir a vida do motor pela degradação do seu sistema de isolamento.

O cabo e o motor podem ser considerados um circuito ressonante excitado pelos pulsos retangulares da tensão modulada gerada pelo variador de velocidade. Assim, as sobretensões são determinadas, basicamente, pelos seguintes fatores: tempo de subida dos pulsos de tensão, tempo mínimo entre pulsos, comprimento do cabo de ligação do motor, e frequência de comutação do variador.

\subsection{Tempo de subida}

Para subir do valor mínimo até ao valor máximo, a tensão PWM requer um certo tempo, denominado de tempo de subida ("rise time"). Os avanços no desenvolvimento dos semicondutores de potência estão a tornar esses tempos cada vez menores e com grande repetibilidade, resultado do crescimento da frequência de comutação dos mesmos. Como consequência a(s) primeira(s) espira(s) da primeira bobina de cada fase fica(m) submetida(s) a um valor de tensão elevado e repetitivo, originando-se uma maior diferença de potencial entre espiras o que degrada mais rapidamente o isolamento dos enrolamento do motor, pelo que este deve possuir características dielétricas reforçadas.
Devido às características indutivas e capacitivas dos enrolamentos do motor, ocorre um amortecimento do pulso nas bobinas subsequentes

\subsection{Comprimento do cabo}

Em muitas aplicações industriais o conversor e o motor têm de estar separados, algumas dezenas ou mesmo centenas de metros. Devido à natureza distribuída da impedância dos cabos, especialmente os mais longos, estes podem apresentar, em função do tempo de subida do pulso de tensão, da frequência de comutação e do seu comprimento, um comportamento igual ao de uma linha de transmissão onde surgem ondas de tensão incidente e refletia [11].

Os pulsos de tensão deslocam-se a uma velocidade aproximadamente igual a metade da velocidade da luz $(150-200 \mathrm{~m} / \mu \mathrm{s})$. Se um pulso de tensão demorar mais do que um terço do tempo de subida a percorrer o cabo, desde o variador ao motor, ocorrerá uma reflexão total aos terminais deste e a amplitude da tensão irá aproximadamente duplicar [11].

É importante salientar que com os cada vez menores tempos de subida dos conversores modernos, um comprimento de cabo relativamente pequeno pode já apresentar este comportamento. De acordo com [12] as sobretensões começam aparecer em cabos a partir de, aproximadamente, $3 \mathrm{~m}$ podendo chegar a 2 vezes o valor da tensão da fonte para comprimentos de cabo de $15 \mathrm{~m}$, e a valores superiores a esse, para comprimentos de cabo acima de $120 \mathrm{~m}$.

Na Figura 3 pode-se observar o valor da sobretensão de um cabo com $30 \mathrm{~m}$ de comprimento e uma impedância característica por metro: $\mathrm{Rc}=20 \mathrm{~m} \Omega / \mathrm{m}, \mathrm{Lc}=1.18 \mu \mathrm{H} / \mathrm{m}$ e Cc $=32.8 \mathrm{pF} / \mathrm{m}$, alimentado por um pulso de tensão com uma amplitude de $600 \mathrm{~V}$ e um tempo de subida $\mathrm{tr}=0.1 \mu \mathrm{s}$.

Confirma-se o pressuposto inicial de que a amplitude da tensão iria aproximadamente duplicar. 

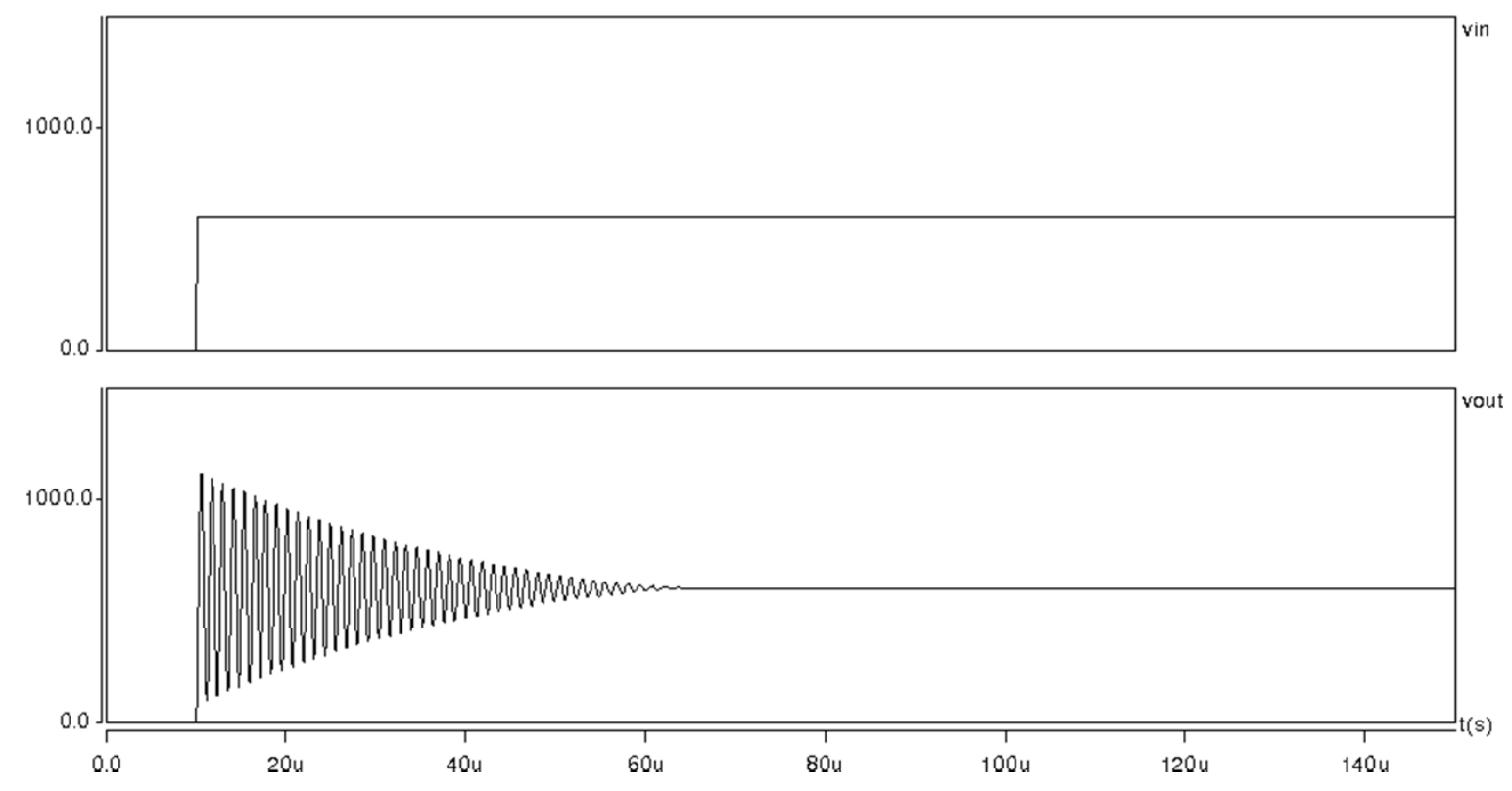

Figura 3. Tensão de entrada e de saída de um cabo elétrico alimentado com um pulso de tensão

Por forma a minimizar estas sobretensões podem ser implementados diferentes tipos de soluções, nomeadamente a introdução de uma bobina em série com o variador e o motor. Esta bobina limita a corrente, filtra a tensão PWM e reduz o ruído elétrico. Contudo, dada a elevada indutância necessária, afeta o desempenho dinâmico do conjunto, é volumosa e cara comparativamente com outras soluções.

Uma segunda solução é o já referido reforço do isolamento dos enrolamentos do motor e também a implementação de filtragem [13]. A filtragem pode utilizar diversas topologias e ser implementada do lado do variador ou do motor.

\subsection{Efeito Corona}

Dependendo da qualidade/homogeneidade do sistema de impregnação, o material impregnante pode conter bolhas de ar (vazios), que podem levar, em conjugação com as sobretensões ao aparecimento de Descargas Parciais que por sua vez podem levar ao rompimento do isolamento entre espiras. Um fenómeno complexo decorrente do efeito Corona. Este efeito resulta do campo elétrico criado pela diferença de potencial entre condutores adjacentes.
Se for estabelecido um campo elétrico suficientemente alto (mas abaixo da tensão de rutura do material isolante), a rigidez dielétrica do ar pode ser rompida, e o oxigênio (O2) é ionizado em ozono (03). O ozono é altamente agressivo e ataca os componentes orgânicos do isolamento dos enrolamentos, deteriorando-os. Para que isso ocorra, o potencial nos condutores precisa exceder um valor limiar denominado CIV (Corona Inception Voltage), que é a rigidez dielétrica do ar "local" (dentro da bolha). O CIV depende do projeto do enrolamento, do tipo de isolamento, da temperatura, de características superficiais e da humidade.

A erosão resulta na diminuição da espessura do material isolante, acarretando progressivas perdas de propriedades dielétricas, que acabarão por levar à falha do isolamento.

\subsection{Tempo Mínimo entre Pulsos Consecutivos e Frequência de Comutação}

Tal como o nome da modulação PWM sugere, o valor eficaz da tensão que é aplicada ao motor é controlado por variação da largura dos pulsos e pelo tempo entre eles. Acontece porem que o efeito de sobretensão é agravado quando o tempo entre os pulsos é mínimo. Condição que ocorre durante regimes transitórios, como aceleração e desaceleração do motor. 
Se o tempo entre pulsos for menor que 3 vezes o período ressonante do cabo (tipicamente 0,2 a $2 \mu \mathrm{s}$ para cabos industriais), ocorrerá um acréscimo na sobretensão.

Associada aos efeitos originados pelo tempo de subida e tempo mínimo entre pulsos consecutivos, está a frequência com que os mesmos são produzidos. Atualmente são já comuns frequências de comutação na ordem dos $20 \mathrm{kHz}$. Porém, há estudos que indiciam que quanto maior for a frequência de comutação mais rápida será a degradação do isolamento dos enrolamentos. A relação de dependência entre o tempo de vida útil do isolamento e a frequência de comutação não é uma relação simples.

Resultados experimentais mostram que para frequências de comutação menores, ou iguais, a $5 \mathrm{kHz}$ a probabilidade de falha do isolamento é diretamente proporcional à frequência de comutação, enquanto que para frequências de comutação maiores que $5 \mathrm{kHz}$ a probabilidade de falha do isolamento é diretamente proporcional ao quadrado da frequência de comutação.

Por outro lado, o aumento da frequência de comutação melhora o conteúdo harmónico da tensão injetada no motor, tendendo, dessa forma, a melhorar o desempenho do motor em termos de temperatura e ruído.

\section{Corrente de Circulação nos Rolamentos}

O problema da tensão/corrente induzida no eixo gravou se com o advento dos variadores de velocidade. A soma vetorial instantânea das três fazes da tensão PWM de saída do variador de velocidade não é igual a zero, mas igual a um potencial elétrico de alta frequência relativamente a um ponto comum de referência, usualmente a terra ou o polo negativo do barramento $D C$, denominada, por isso de tensão de modo comum. Havendo capacidades parasitas do motor para a terra, ocorre a circulação de uma corrente de modo comum indesejada que atravessa o rotor, eixo, rolamento e tampa aterrada.

Portanto, as causas de tensão induzida no eixo devido aos variadores de velocidade somam-se àquelas intrínsecas ao motor (por exemplo, desbalanceamento eletromagnético Causado por assimetria) e que também provocam a circulação de corrente nos rolamentos.

Estas correntes desgastam as esferas e a pista dos rolamentos, dando origem a pequenos furos, que começam a sobrepor-se e, e que com o passar do tempo promovem a formação de sulcos (Figura 5), reduzindo a vida útil dos rolamentos e podendo mesmo provocar a falha do motor.

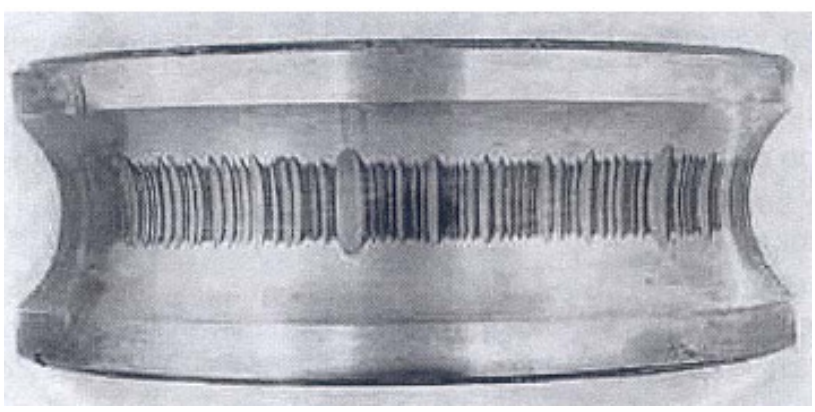

Figura 5. Pista de rolamento danificada devido à circulação de corrente elétrica (fonte [4]).

Estes efeitos podem ser mitigados utilizando rolamentos isolados e introduzindo escovas de aterramento entre o rotor e a carcaça do motor por intermédio de uma escova deslizante de grafite.

\section{Conclusões}

O rápido desenvolvimento da eletrónica de potência tem permitido que os motores de indução, tradicionalmente acionados a velocidade constante, sejam utilizados com sucesso em aplicações de velocidade variável. Nestes casos, o motor é alimentado por um variador de velocidade.

Estes sistemas apresentam grandes vantagens tanto energéticas quanto económicas, quando comparado com outras soluções existentes para aplicações industriais de velocidade variável. No entanto, o uso do variador traz consequências para o motor, fazendo com que os fabricantes de motores precisem de estar atentos. 


\section{Referências}

[1] C. Choochuan., "A survey of output filter topologies to minimize theimpact of pwm inverter waveforms on three-phase ac induction motors," em he 7th International Power Engineering Conference - IPEC 2005, 2005.

[2] E. N. H. a. H. Roehrdanz, "Losses in three-phase induction machines fed by pwm converter," IEEE Transactions on Energy, 2001.

[3] T. C. G. a. A. C. S. Carlos A. Hernandez-Aramburo, "Estimating rotational iron losses in an induction machine," IEEE Transactions on Magnetics, vol. 39, 2003.

[4] H. N. K.-H. H. J.-P. H. a. D.-H. H. Jeong-Jong Lee, "Loss distribution of three-phase induction motor fed by pulsewidth-modulated inverter," EEE Transactions on Magnetics, vol. 40, 2004.

[5] A. M. J. S. S. B. a. G. L. S. Mike Melfi, "Effect of surge voltage risetime on the insulation of low-voltage machines fed by pwm converters," IEEE Transactions on Industry Applications, vol. 34, p. 766-775, 1998.

[6] S. Chen, T. Lipo e D. Novotny, "Circulating type motor bearing current in inverter drives," em Thirty-First IAS Annual Meeting, IAS '96, 1996.

[7] S. Ueda, K. Honda, T. Ikimi, M. Hombu e A. Ueda, "Magnetic noise reduction technique for an AC motor driven by a PWM inverter," IEEE Transactions on Power Electronics, vol. 6, pp. 470-475, Jul 1991.

[8] N. Hashemi, R. Lisner e D. Holmes, "Acoustic noise reduction for an inverter-fed three-phase induction motor," em 39th IAS Annual Meeting Industry Applications Conference, 2004.

[9] WEG Equipamentos Elétricos S.A., "Guia Técnico Motores de indução alimentados por inversores de frequência PWM," Jaraguá do Sul, 2009.

[10] H. G. G. M. Waldiberto de Lima Pires, “Minimização de Perdas em Motores de Indução Alimentados Por Conversores de Frequência - Solução Fluxo Ótimo," Jaraguá do Sul - Barsil, 2006.
[11] P. E. a. W. G. A. von Jouanne, "Application issues for pwm adjustable speed ac motor drives," IEEE Industry Application Magazine, 1996.

[12] NEMA Standards Publication, "Application Guide for AC Adjustable Speed Drive Systems," National Electrical Manufacturers Association, Rosslyn, 2007.

[13] P. T. Finlayson, "Output filters for pwm drives with induction motors," IEEE Industry Applications Magazine, p. 46-52, 1998.

\section{Notas soltas:}

\begin{tabular}{|c|c|c|c|}
\hline \multicolumn{2}{|c|}{ Grandeza física } & \multicolumn{2}{|c|}{ Unidade } \\
\hline Denominação & Símbolo & Denominação & Símbolo \\
\hline Capacidade & C & Farad & $\mathrm{F}$ \\
\hline Carga elétrica & Q & Coulonb & C \\
\hline Comprimento & L & metro & $\mathrm{m}$ \\
\hline $\begin{array}{l}\text { Corrente } \\
\text { elétrica }\end{array}$ & 1 & Ampére & A \\
\hline $\begin{array}{c}\text { Densidade de } \\
\text { corrente }\end{array}$ & J & Ampére $/ \mathrm{m}^{2}$ & $\mathrm{~A} / \mathrm{m}^{2}$ \\
\hline Energia & $\mathrm{E}$ & Joule & J \\
\hline $\begin{array}{c}\text { Fluxo } \\
\text { magnético }\end{array}$ & $f$ & Weber & $\mathrm{Wb}$ \\
\hline Força & $\mathrm{F}$ & Newton & N \\
\hline Frequência & $\mathrm{F}$ & Hertz & $\mathrm{Hz}$ \\
\hline Impedância & z & Ohm & $\Omega$ \\
\hline Indutância & L & Henry & $\mathrm{H}$ \\
\hline $\begin{array}{l}\text { Potência } \\
\text { elétrica }\end{array}$ & $P$ & Watt & W \\
\hline Pressão & $P$ & Pascal & $\mathrm{Pa}$ \\
\hline $\begin{array}{l}\text { Resistência } \\
\text { elétrica }\end{array}$ & $\mathrm{R}$ & Ohm & $\Omega$ \\
\hline Resistividade & $r$ & Ohmxmetro & $\Omega \times m$ \\
\hline $\begin{array}{l}\text { Tensão } \\
\text { elétrica }\end{array}$ & V & Volt & V \\
\hline
\end{tabular}

